\title{
The Capturing of Space Debris with a Spaceborne Multi- fingered Gripper
}

\author{
Yin Zhang ${ }^{1}$ and Qiang Zhan ${ }^{2, a}$ \\ ${ }^{1}$ Robotic institute, Beihang University, 100191 Beijing, China \\ 2 Robotic institute, Beihang University, 100191 Beijing, China
}

\begin{abstract}
With the massive launching of spacecraft, more and more space debris are making the low Earth orbit (LEO) much more crowded which seriously affects the normal flight of other spacecrafts. Space debris removal has become a very urgent issue concerned by numerous countries. In this paper, using SwissCube as a target, the capturing of space debris with a spaceborne four-fingered gripper was studied in order to obtain the key factors that affect the capturing effect. The contact state between the gripper fingers and SwissCube was described using a defined contact matrix. The law of momentum conservation was used to model the motion variations of the gripper and SwissCube before and after the capturing process. A zero-gravity simulation environment was built using ADAMS software. Two typical kinds of capturing processes were simulated considering different stiffness of fingers and different friction conditions between fingers and SwissCube. Comparisons between results obtained with the law of momentum conservation and those from ADAMS simulation show that the theoretical calculations and simulation results are consistent. In addition, through analyzing the capturing process, a valuable finding was obtained that the contact friction and finger flexibility are two very important factors that affect the capturing result.
\end{abstract}

\section{Introduction}

Space debris is the collection of defunct man-made objects in orbit around the Earth, which includes old satellites, spent rocket stages, fragments, erosion, and collisions, et al [1]. A lot of space debris has been generated during the past 60 years of human space activities. Most of the space debris spreads within the Low Earth Orbit (LEO) region, resulting in the density of space debris on orbit is approaching the safety threshold [2,3]. It is well recognized that the existence of space debris seriously threatens the safety of human space activities and the orbiting spacecrafts, so it is essential for the whole world to remove the LEO space debris.

The space environment is very complex with micro-gravity, high vacuum, strong radiation and weak illumination. Space debris belongs to non-cooperative target and has different shapes and sizes. It's significantly challenging to capture and remove space debris. Space agencies in many countries have proposed some projects and plans on space debris removal, which can be broadly categorized as follows: 1) "Robotic Arm \& Gripper", such as the FREND project [4], the DEOS and the EPOS project [5] and the e.Deorbit project [6];2) "Flexible Net", such as the e.Deorbit project [6]; 3)

\footnotetext{
${ }^{a}$ Corresponding author : qzhan@buaa.edu.cn
} 
"Harpoon", such as the e.Deorbit project [6]; 4) "Electro-dynamic Tether", such as the ProSEDS plan [7]. Some other methods have also been proposed and studied, such as the laser method [8], etc. Although many studies have been done on space debris removal, however, to find a safe, reliable and efficient method still has a long way to go.

In order to explore and verify the key technologies of the LEO space debris removal, EPFL (École Polytechnique Fédérale de Lausanne) and Swiss Space Center proposed the "CleanSpace One, CSO" plan $[9,10]$. Based on the information communicated with EPFL, we have studied the capturing of target SwissCube with a four-fingered spaceborne gripper in order to check the reliability and factors influencing the grasping scheme.

\section{Spaceborne gripper capturing scheme}

\subsection{Brief introduction of Swiss Cube}

The capturing target SwissCube was launched in 2009 by Swiss Space Center. Some of its main characteristic parameters are provided in Table 1. It was originally designed working for 4-12 months, however, it has been working for over 7 years to date. Some components have begun to age and therefor it is chosen as the experimental target to remove from the orbit [9].

Table 1. Characteristics of SwissCube.

\begin{tabular}{ll}
\hline Parameters & Values \\
\hline Dimensions (Length $\times$ Width $\times$ Height $) /$ & \\
$\mathrm{mm} \times \mathrm{mm} \times \mathrm{mm}$ & $113.50 \times 100.00 \times 100.00$ \\
Mass $/ \mathrm{kg}$ & 1.00 \\
The maximum rotation speed $/ \mathrm{deg} \cdot \mathrm{s} \mathrm{s}^{-1}$ & 50.00 \\
The maximum moment of inertia $/ \mathrm{kg} \cdot \mathrm{m}^{2}$ & $2.45 \times 10^{-3}$ \\
\hline
\end{tabular}

\subsection{Spaceborne gripper capturing system}

In this paper, we studied a spaceborne gripper system that regards SwissCube as the target for capturing. Compared with the flexible net and other removal methods, this gripper capturing system can conduct the capturing and releasing process more easily, and it can repeat the removal tasks. According to the robot grasping theory, the number of fingers is typically greater than or equal to three. Because the cracks between fingers are larger of the three-fingered gripper, the possibility of target escape is larger. The structure of the five-fingered gripper is complex and difficult to control. Therefore, considering factors such as structural complexity, grasping stability and others, we select four-fingered gripper as the spaceborne capturing device scheme. The gripper is composed of a base and four arc-shaped fingers. The gripper can adjust its own posture according to the moving state of SwissCube by controlling the closing and opening of the fingers, the gripper can realize the capturing and releasing of the target. As we mainly focus on the capturing process, we just consider the system model comprising mother-satellite, spaceborne gripper, and SwissCube.

\section{Modeling and analysis of the capturing process}

The spaceborne capturing system will be nearly on the same orbit as the SwissCube after completing the tracking and closing steps. The angular velocity difference around the Earth could be ignored. When the distance between the capturing system and SwissCube is small, the linear speed difference between the two objects could be also neglected. Therefore, the final capturing process could be simplified as one where a spaceborne gripper captures SwissCube in a zero gravity environment.

\subsection{Description of the contact status}




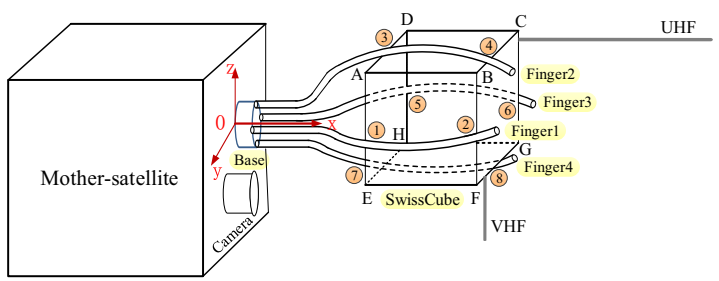

Figure 1. The grasping model of spaceborne gripper capturing SwissCube.

Fig. 1 shows the grasping model. SwissCube has two antennas, six feature planes, and twelve different edges. When the fingers contact with SwissCube, contact forces will be applied on SwissCube through the contact points and a group of point contact constraints will be formed. The maximum number of contact points between one finger and one edge of SwissCube is two. We defined a contact matrix $\boldsymbol{M}_{F_{i}, L_{j}}$ to describe the contact status among fingers and SwissCube,

$$
\boldsymbol{M}_{F_{i}, L_{j}}=\left[\delta_{i, j}\right] \quad \mathrm{i}=1, \ldots, 4, \mathrm{j}=1, \ldots, 12
$$

Where, $F_{i}$ represents the $i$ th finger of the gripper, $F_{i}=$ Finger $i$; $L_{j}$ represents the $j$ th edge of SwissCube, $L_{1}=A B, L_{2}=B C, L_{3}=C D, L_{4}=D A, L_{5}=E F, L_{6}=F G, L_{7}=G H, L_{8}=H E, L_{9}=A E, L_{10}=B F$, $L_{11}=C G, L_{12}=D H$. $\delta_{i, j}$ denotes the contact status between the $i$ th finger and the $j$ th edge. If the $i$ th finger contacts with the $j$ th edge, $\delta_{i, j}=1$; else, $\delta_{i, j}=0$. When SwissCube is moving in the envelope region, different contact points will be formed and the contact matrix $\boldsymbol{M}_{F_{i}, L_{j}}$ will be different.

\subsection{Theoretical analyses of the capturing process}

The complex contact and collision process can be seen as an impulse effect. We defined a contact force matrix $\boldsymbol{F}_{F_{i}, L_{j}}$ to describe the contact forces between the fingers and SwissCube,

$$
\boldsymbol{F}_{F_{i}, L_{j}}=\left[f_{i, j}\right] \mathrm{i}=1, \cdots, 4, \mathrm{j}=1, \cdots, 12
$$

Where, $f_{i, j}$ denotes the contact force between the $i$ th finger and the $j$ th edge of SwissCube. If the $i$ th finger contact with the $j$ th edge, $\delta_{i, j}=1$ and $f_{i, j} \neq 0$; else, $\delta_{i, j}=0$ and $f_{i, j}=0$.

In the space environment, all the linear momentum and angular momentum of the whole system are both constants. We can utilise the law of momentum conservation to analyze the motion variation of the system before and after the capturing process. The initial linear momentum and the angular momentum of the whole system are shown in Eq. 3 and Eq. 4 respectively.

$$
\begin{gathered}
\boldsymbol{P}_{I}=\boldsymbol{P}_{M}+\boldsymbol{P}_{S}=\sum_{i=1}^{n} m_{i} \boldsymbol{v}_{i}+m_{S} \boldsymbol{v}_{S} \\
\boldsymbol{L}_{I}=\boldsymbol{L}_{M}+\boldsymbol{L}_{S}=\sum_{i=1}^{n}\left(\boldsymbol{r}_{\mathrm{i}} \times m_{i} \boldsymbol{v}_{i}\right)+\boldsymbol{r}_{S} \times m_{S} \boldsymbol{v}_{S}
\end{gathered}
$$

Where $\boldsymbol{P}_{M}$ and $\boldsymbol{L}_{M}$ denote the linear and angular momentum of the mother-satellite with gripper respectively, $m_{i}, \boldsymbol{v}_{i}$ and $\boldsymbol{r}_{i}$ denote the mass, the line speed, and the radius vector of each corresponding components. $\boldsymbol{P}_{S}, \boldsymbol{L}_{S}, m_{S}, \boldsymbol{v}_{S}$ and $\boldsymbol{r}_{S}$ denote the linear momentum, the angular momentum, the mass, the line speed and the radius vector of SwissCube respectively.

The initial line speed and angular velocity of the mother-satellite are both set to 0 . The initial line speed and angular velocity of each finger are set to 0 and $\omega_{f_{k}}$. The initial line speed and angular velocity of SwissCube are set to 0 and $\boldsymbol{\omega}_{S_{Z}}$. Thus, $\boldsymbol{P}_{I}=0$ and $\boldsymbol{L}_{I}=\sum_{k=1}^{4} J_{f_{k}} \boldsymbol{\omega}_{f_{k}}+J_{S} \boldsymbol{\omega}_{S_{z}}$, where, 
$J_{f_{k}}$ and $J_{S}$ denote the inertia moment of each finger and that of SwissCube respectively. Since the four fingers are even-distributed along one circle, so $\sum_{k=1}^{4} J_{f_{k}} \boldsymbol{\omega}_{f_{k}}=0$, and $\boldsymbol{L}_{I}=J_{S} \boldsymbol{\omega}_{S_{Z}}$.

We use $\boldsymbol{P}_{E}$ and $\boldsymbol{L}_{E}$ to denote the final linear and angular momentum of the whole system respectively. According to the law of momentum conservation, the following equation is obtained,

$$
\left[\begin{array}{l}
\boldsymbol{P}_{I} \\
\boldsymbol{L}_{I}
\end{array}\right]=\left[\begin{array}{l}
\boldsymbol{P}_{E} \\
\boldsymbol{L}_{E}
\end{array}\right]=\left[\begin{array}{c}
0 \\
J_{S} \boldsymbol{\omega}_{S_{Z}}
\end{array}\right]
$$

The final linear speed is zero, $\boldsymbol{v}_{E}=0$, and the final angular velocity $\boldsymbol{\omega}_{E}$ is shown in Eq. 6 .

$$
\boldsymbol{\omega}_{E}=\left[\begin{array}{lll}
\boldsymbol{\omega}_{E x} & \boldsymbol{\omega}_{E y} & \boldsymbol{\omega}_{E z}
\end{array}\right]^{T}=\left[\begin{array}{lll}
0 & 0 & J_{S} \boldsymbol{\omega}_{S_{z}}
\end{array}\right]^{T} / J_{E}
$$

Where $\boldsymbol{\omega}_{E x}, \boldsymbol{\omega}_{E y}$ and $\boldsymbol{\omega}_{E z}$ denote the angular velocity about the axes $x, y$ and $z$ respectively, $J_{E}$ denotes the inertia moment of the whole system including SwissCube.

We use the following values to explain the changes before and after the capturing process, namely, $\sum_{i=1}^{n} m_{i}=30 \mathrm{~kg}, m_{\mathrm{S}}=1 \mathrm{~kg}, J_{S}=2.45 \times 10^{-3} \mathrm{~kg} \cdot \mathrm{m}^{2}$ (shown in Table 1), $J_{E}=0.53483 \mathrm{~kg} \cdot \mathrm{m}^{2}$, $\boldsymbol{\omega}_{S_{Z}}=50 \mathrm{deg} / \mathrm{s}$. Thus, we can obtain the following results, $\boldsymbol{\omega}_{E x}=\boldsymbol{\omega}_{E y}=0$ and $\boldsymbol{\omega}_{E z}=0.229 \mathrm{deg} / \mathrm{s}$.

It indicates that if the gripper can capture SwissCube successfully, the gripper, the mother-satellite and SwissCube will rotate about their common axis at a same small angular velocity together.

\section{Simulation and analysis of the capturing process}

ADAMS software was used to build a zero-gravity simulation environment, some parameters are set as follows: the mass of the mother-satellite and SwissCube are $30 \mathrm{~kg}$ and $1 \mathrm{~kg}$ respectively, the initial angular velocity of SwissCube is $50 \mathrm{deg} / \mathrm{s}$. If the contact belongs to frictionless point contact, it is difficult to capture SwissCube successfully. We mainly focus on the other two typical capturing cases with different stiffness of fingers and different friction conditions. In order to display the simulation results conveniently, we adopt the following abbreviations: MS-Microsatellite, SC-SwissCube, $P$ Position, V-Velocity, A-Acceleration, AV-Angular Velocity, AA-Angular Acceleration.

\subsection{Simulation and analysis of "Rigid fingers \& Contact friction"}

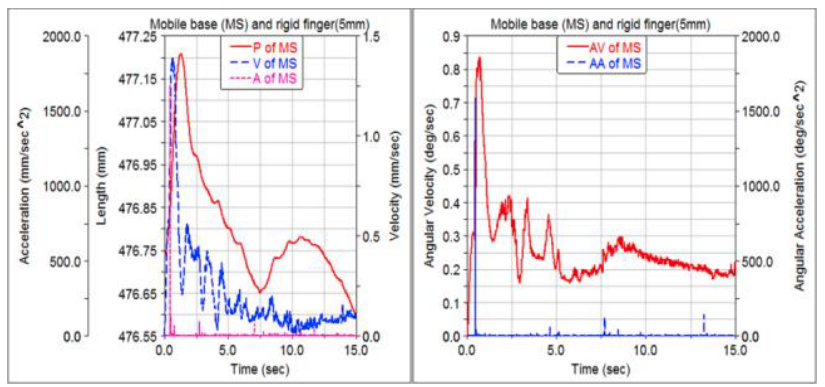

Figure 2. Motion variations of mother-satellite

Fig. 2 and Fig. 3 show the motion variations of the mother-satellite and SwissCube after capturing when the acting force is a force with friction. From these figures, we can find that when the fingers first contact with SwissCube, the mother-satellite has a notable acceleration, its line speed reaches a high value in a short time and then gradually decreases to zero. The mother-satellite deviates from its initial position slightly and then gradually returns to the initial position. The final angular velocity of SwissCube and the mother-satellite will be a same smaller one $(\approx 0.2 \mathrm{deg} / \mathrm{s})$ about their common 
equivalent axis. These results are consistent with those obtained from the theoretical analysis. Finally, the angular acceleration will eventually be zero. The gripper can capture SwissCube successfully.
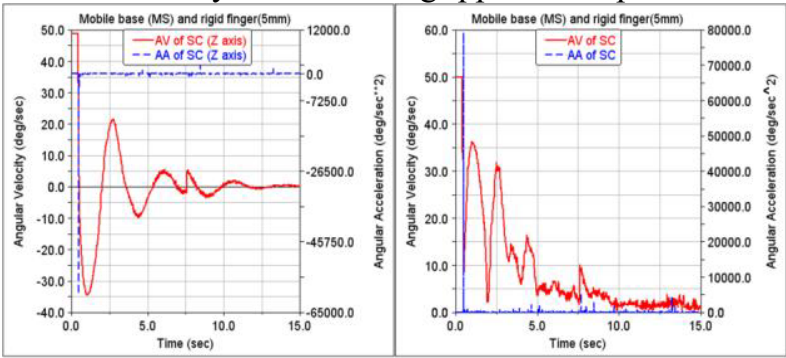

Figure 3. Motion variations of SwissCube

Fig. 4(a) shows the initial state of the whole system before capturing. When the contact belongs to point contact with friction, some kinetic energy of SwissCube could be "consumed" and converted into other forms of energy. SwissCube moves and rotates in the envelope region formed by the fingers and it gradually reaches a stable state (see Fig. 4(b)). As there exists motion coupling between the mother-satellite and SwissCube, the final angular velocity of SwissCube and the mother-satellite will be not zero, but a same smaller one about their common equivalent axis.

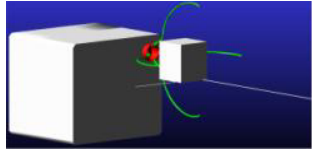

(a) Initial state

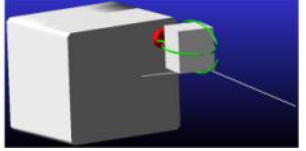

(b) After capturing

Figure 4. Capturing result with contact friction and the fingers are rigid

\subsection{Simulation and analysis of "Flexible fingers \& Contact friction"}

In this section, we'll explore the capturing characteristic of the system when the fingers are flexible bodies and there exists contact friction between fingers and SwissCube. It means that the rigid fingers are converted to elastic bodies using FEA software and replaced by flexible fingers in ADAMS software. Thus, a whole system consists of rigid parts and flexible fingers is built. Flexible fingers can deform under force. The contact between the fingers of the gripper and SwissCube also belongs to point contact with friction.

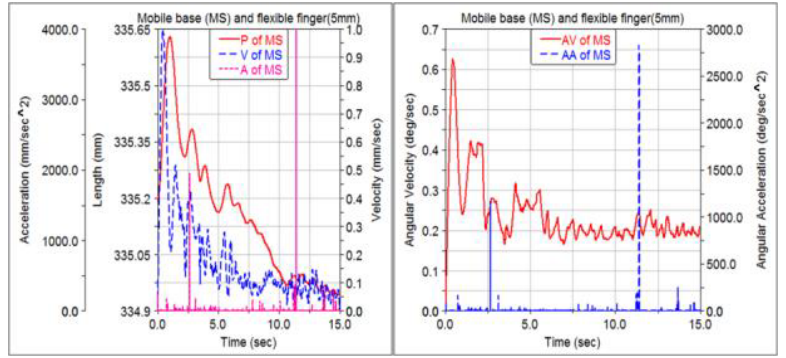

Figure 5. Motion variations of mother-satellite

When the simulation is completed, we get the motion variations of the mother-satellite and SwissCube (shown in Fig. 5 and Fig. 6). Because there exists motion coupling between the fingers and SwissCube, the mother-satellite deviates from its initial position slightly at a small line speed and it rotates about its own axis. Through comparing with the motion variations of the mother-satellite and SwissCube when the fingers are rigid bodies, we can find that the flexible fingers can make the capturing process more smooth and continuous than the rigid fingers, and the whole system can reach a stable state more quickly. This is because when the fingers are flexible bodies, some kinetic energy of SwissCube could be "consumed" and converted into other forms of energy, so the mothersatellite and SwissCube can reach the co-speed $(\approx 0.2 \mathrm{deg} / \mathrm{s})$ rotation state more smoothly and quickly. 


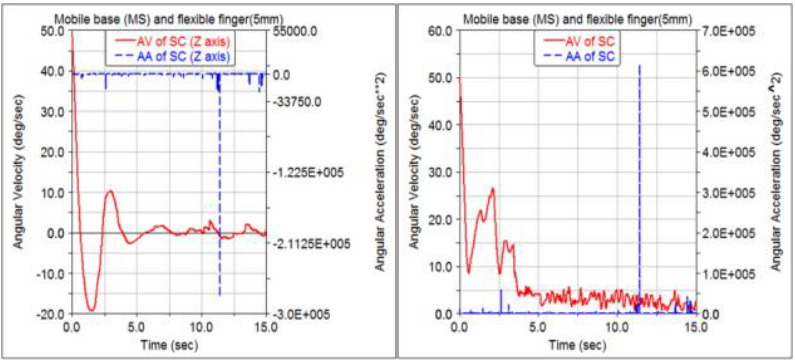

Figure 6. Motion variations of SwissCube

\section{Conclusions}

In this paper, we studied the capturing process and its influencing factors when a four-fingered spaceborne gripper captures a target space debris SwissCube. We can conclude the following:

1. Considering the structural complexity, control difficulty, grasping stability and other factors, the four-fingered gripper is an ideal capturing device scheme. If this spaceborne gripper can approach the approximate capturing region, it can capture the space debris target successfully.

2. Contact friction is an important and essential factor affecting the space debris capturing effect. It's helpful to "consumed" the kinetic energy of the object and converted it into other forms of energy. When designing a capturing device, the contact friction should be considered.

3. The flexibility of finger is another significant factor affecting the capturing effect. Flexible fingers have better performance than rigid fingers, it's helpful to make the capturing more smooth and quickly. When designing a capturing device, the flexibility of the gripper should be taken into account.

\section{Acknowledgements}

This work was supported by the Fundamental Research Funds for the Central Universities (YWF-14JXXXY-025).

\section{References}

1. M.H. Shan, J. Guo and E. Gill, Review and comparison of active space debris capturing and removal methods, Prog Aerosp Sci. 80 (2016) 18-32.

2. J.-C. Liou, N.L. Johnson and N.M. Hill, Controlling the growth of future LEO debris populations with active debris removal, Acta Astronaut. 66 (2010) 648-653.

3. C. Bonnal, J.M. Ruault and M.C. Desjean, Active debris removal: recent progress and current trends, Acta Astronaut. 85 (2013) 51-60.

4. G. Creamer, The SUMO/FREND project: technology development for autonomous grapple of geosynchronous satellites, Adv Astronaut Sci. 128 (2007) 895-909.

5. T. Boge, T. Wimmer, O. Ma and M. Zebenay, EPOS-A robotics-based hardware-in-the-loop simulator for simulating satellite RvD operations. In: 10th International symposium on artificial intelligence, robotics and automation in space, Sapporo, Japan, 2010.

6. J.A.F. Deloo, Analysis of the rendezvous phase of e.Deorbit: guidance, communication and illumination. PhD Thesis, Delft University of Technology, NL, 2015.

7. S. Kawamoto, T. Makida, F. Sasaki, et al, Precise numerical simulations of electrodynamic tethers for an active debris removal system, Acta Astronaut. 59 (2006) 139-148.

8. C.R. Phipps, K.L. Baker, S.B. Libby, et al, Removing orbital debris with lasers, Adv Space Res. 49 (2012) 1283-1300.

9. M. Richard, L. Kronig, F. Belloni, et al, Uncooperative rendezvous and docking for MicroSats, In: 6th International conference on recent advances in space technologies, Istanbul, Türkiye, 12-14 June 2013.

10. O.A. Araromi, I. Gavrilovich, J. Shintake, et al, Rollable multisegment dielectric elastomer minimum energy structures for a deployable microsatellite gripper, IEEE/ASME Transactions on Mechatronics. 20 (2015) 438-446. 